\title{
ÚLCERA DUODENAL PERFURADA EM PACIENTE SUBMETIDO A BYPASS GÁSTRICO EM Y-DE-ROUX
}

\author{
Mariangela Latini de MIRANDA ${ }^{1}$
}

Paula Lutfalla PESSÔA²

\begin{abstract}
${ }^{1}$ Médica Residente de Cirurgia Geral no Hospital Márcio Cunha - Ipatinga - MG - mariangela_latini@ @otmail.com
2Médica Residente de Cirurgia Geral no Hospital Márcio Cunha - Ipatinga - MG - paulalutfalla@ hotmail.com
\end{abstract}

Recebido em: 24/07/2016 - Aprovado em: 02/12/2017 - Disponibilizado em: 30/12/2017

\begin{abstract}
RESUMO:
A obesidade é uma doença relevante nos dias atuais e de grande impacto na saúde da população mundial, sendo o Bypass Gástrico em Y-de-Roux amplamente utilizada no tratamento cirúrgico da obesidade mórbida, entretanto esta técnica não é isenta de complicações. A úlcera duodenal perfurada no segmento excluso em pacientes submetidos ao Bypass Gástrico em Y-de-Roux é uma patologia rara sendo encontrado na literatura apenas 21 casos no mundo. Existem evidências que o segmento excluso mantem-se produtor de ácidos gástricos sem a neutralização do alimento, Este relato de caso trata-se de uma paciente com história de Bypass Gástrico em Y-de-Roux hà 5 anos, que apresentou quadro de dor abdominal após uso prolongado de AINE, sendo encaminhada para videolaparoscopia. Foi diagnosticado úlcera duodenal perfurada em segmento excluso e o tratamento definitivo procedeu em seguida, sendo realizado ulcerorrafia e omentoplastia.

Palavras-chave: Obesidade mórbida. Bypass gástrico em Y-de-Roux laparoscópico. Úlcera duodenal perfurada. Úlcerorrafia. Reparo laparoscópico.
\end{abstract}

\begin{abstract}
:
Obesity is an important disease in the present day and of great impact on the health of the world population, being the Roux-en-Y gastric by-pass widely used in the surgical treatment of morbid obesity, however this technique is not without complications. The perforated duodenal ulcer in the excluded segment in patients undergoing Roux-en-Y gastric by-pass is a rare disease being found worldwide in the literature only 21 cases. There is evidence that the excluded segment keeps producing gastric acid without food neutralization. This case report it is a patient with a history of Roux-en-Y gastric by-pass 5 years before, who presented abdominal pain after prolonged use of NSAIDs, being referred to laparoscopy. It was diagnosed perforated duodenal ulcer in excluded segment and thereafter definitive treatment, being fulfilled ulcerorrafia and omentoplasty.
\end{abstract}

Keywords: Morbid obesity. Laparoscopic Roux-en-Y gastric by-pass. Duodenal ulcer perforation. Ulcerorrafia. Laparoscopic repair.

\section{Introdução}

A obesidade é uma doença de grande importância no mundo, atinge aproximadamente 2 bilhões de pessoas e sua prevalência atualmente é crescente. Ela está associada a múltiplas comorbidades como diabetes tipo 2, hipertensão arterial, dislipidemias e apneia do sono (GARRIDO JÚNIOR, 2006). Os critérios para indicação de tratamento cirúrgico compreendem em pacientes com Índice de Massa Corpórea $\left(\right.$ IMC) $>40 \mathrm{~kg} / \mathrm{m}^{2}$ ou IMC $>35 \mathrm{~kg} / \mathrm{m}^{2}$ com a presença de comorbidades. Embora o grupo de pacientes com indicação cirúrgica são a minoria na obesidade, representado por apenas $10 \%$ da população obesa, o mesmo se encontra em constante ascensão (GARRIDO JÚNIOR, 2006; MONKHOUSE et al., 2009).

O número de cirurgias para o tratamento da obesidade vem aumentando 
consideravelmente, já que se mostrou como um método seguro e eficaz na redução de peso e também tratamento das comorbidades, conforme apresentado pelo Instituto Nacional de Saúde de 1996 (NIH-USA) (TINOCO et al., 2002). No Brasil há quatro técnicas aprovadas: Banda Gástrica Ajustável, Duodenal Switch, Gastrectomia Vertical e o Bypass Gástrico em Y-de-Roux (BGYR), sendo essa última a técnica mais usada atualmente por ser mais segura e eficaz (ABESO, 2009).

A úlcera duodenal perfurada em pacientes submetidos a BGYR é uma complicação rara sendo encontrado na literatura apenas 21 casos bem reportados no mundo (ISKANDAR et al., 2014). O tempo entre a cirurgia e a perfuração da úlcera duodenal pode variar entre 20 dias e 12 anos (MACGREGOR et al., 1999), porém a maioria dos casos notificados apontam uma incidência maior no pós-operatório precoce em pacientes que não estavam em uso de Inibidores de Bomba de Prótons (IBP) (ISKANDAR et al., 2014).

A fisiopatologia da ulcerogênese em pacientes submetidos ao BGYR não está bem estabelecida. Vários mecanismos têm sido usados para explicar e acredita-se que a infecção por Helicobacter pylori seja o principal fator responsável pela destruição da barreia mucosa além de aumento da secreção ácida gástrica (KINI; KANNAN, 2011; SIQUEIRA et al., 2007). Outro mecanismo reconhecido é a ausência de neutralização ácido no estômago pelo alimento ingerido, como ocorre em pacientes sem alterações anatômicas, além disso existe um atraso na liberação de bicarbonato, sendo a mucosa exposta ao ácido gástrico e refluxo biliar por um período prolongado (BJORKMAN et al.,1989). As lesões de mucosa também podem ser resultado do uso de drogas não esteroides anti-inflamatórios não esteroides (AINE) ou consumo excessivo de álcool (BJORKMAN et al.,1989).

O diagnóstico da úlcera duodenal em pacientes submetidos a BGYR pode ser desafiador e devemos atentar para pacientes com o histórico cirúrgico de BGYR associado principalmente a dor em abdome superior, mesmo na ausência de pneumoperitônio nos exames de imagem, já que a presença desse é incomum nesse grupo de pacientes (CHARUZI, 1986).

A dor abdominal é uma queixa comum em pós-operatório de BGYR atingindo 15\% a $30 \%$ dos pacientes (GREENSTEIN; O'ROURKE, 2011). Diante disso a úlcera duodenal perfurada no segmento excluso deverá ser um diagnóstico diferencial em pacientes com dor abdominal e histórico cirúrgico de BGYR, mesmo na ausência de pneumoperitôneo. Nesses pacientes a exploração cirúrgica precoce deverá ser cogitada tanto para auxílio diagnóstico quanto terapêutico (ISKANDAR et al., 2014). Este artigo traz o relato de um caso clínico raro 
perante a comunidade científica, de uma complicação incomum do Bypass Gástrico em Y-de-Roux, considerando que esse grupo na maioria das vezes apresenta manifestações incomuns, que devem ser atentadas em unidades de pronto atendimento médico.

\section{Relato de caso}

Uma mulher de 41 anos, deu entrada no pronto-socorro em fevereiro de 2015 com história de dor abdominal hà dois dias, com piora progressiva. Dor inespecífica, mais em região inferior do abdome e flaco direito. Negou alterações urinárias, intestinais, ginecológicas ou febre. Estava em uso de anti-inflamatórios não esteroidais (AINEs) devido pós operatório recente de varicectomia bilateral. Relata ter apresentado episódios isolados de dor em flanco direito, e de epigastralgia com piora ao alimentar-se, desde o ano de 2013. Possui história de cirurgias prévias como colecistectomia videolaparoscópica em outubro de 2010, e pouco antes, em junho de 2010, Bypass Gástrico em Y-de-Roux, onde foi ultilizado a técnica Fobi-Capella, para tratamento de obesidade crônica refratária aos tratamentos instituídos. Nesta época, apresentava peso $96 \mathrm{~kg}$, com estatura de $1,56 \mathrm{~m}$ chegando a um IMC de $40 \mathrm{~kg} / \mathrm{m}^{2}$, não apresentava comorbidades associadas, após cirurgia apresentou redução do seu peso gradativamente, segundo dados do prontuário. Ao exame físico apresentava-se com fáscies de dor, corada, hidratada, eucárdica e eupneica, pressão arterial de 130 por 80 mmHg. Abdome flácido, doloroso a palpação em andar inferior, mais localizado em fossa ilíaca direita, região superior do abdome não apresentava dor, sem sinais de irritação peritoneal, sem massa palpável. Não apresentou alterações nos exames laboratoriais solicitados como creatinina, uréia, amilase, lipase, AST, ALT, exceto por hemograma com anemia e leucocitose discreta, EAS com 8 hemáceas por campo (valor de referência menos de 3 a 5 hemáceas por campo) e PCR no valor de $10 \mathrm{mg} / \mathrm{L}$ (valor de referência $5 \mathrm{mg} / \mathrm{L}$ ). Foi solicitado exame de ultrassonografia abdominal tendo como achados coleção líquida na pelve à direita, com septos internos, medindo 6,9 x 5,5 x $2,5 \mathrm{~cm}$ e com volume estimado de $50 \mathrm{ml}$, além de pequena quantidade de líquido anecóide junto a base do ceco. Com a hipótese diagnóstica de um abdome agudo inflamatório ou hemorrágico, a laparoscopia foi indicada.

$\mathrm{Na}$ exploração inicial laparoscópica, visualizou-se útero, tubas uterinas e ovários normais, foi identificado cerca de $150 \mathrm{ml}$ de líquido amarelado na pelve e goteira parietocólica direita, apêndice cecal hiperemiado e congesto, cólon sigmoide redundante e distendido, sendo realizado apendicectomia e irrigação da cavidade. Em revisão da cavidade observou-se bloqueio inflamatório entre o lobo direito do fígado e colon, em exploração houve saída de pequena 
quantidade secreção biliosa e visualizado perfuração da parede anterior duodenal, realizado rafia da úlcera duodenal. Durante o ato cirúrgico não houve intercorrências, segundo dados coletados. A paciente seguiu para a enfermaria após recuperação anestésica, recebendo alta no $3^{\circ}$ dia pósoperatório, em boas condições clínicas.

\section{Discussão}

Esta paciente do caso relatado apresentava dores abdominais inespecíficas e recorrentes anteriormente, com piora súbita da dor, concentrando em fossa ilíaca direita e uma história de uso prolongado de antiinflamatórios não esteroidais. $\mathrm{O}$ quadro clínico sugeriu abdome agudo de etiologia a esclarecer, sendo a úlcera duodenal perfurada uma das possibilidades. Uma vez que se tratava de uma paciente com alterações anatômicas precedente da cirurgia de Bypass Gástrico em Y-de-Roux, os exames de rotina para abdome agudo em muitos casos não evidenciam sinais sugestivos de úlcera perfurada como de costume em pacientes sem estas alterações anatômicas, sendo na maioria das vezes diagnosticado com a videolaparoscopia.

Em busca do tratamento ideal para a obesidade, várias técnicas foram desenvolvidas sendo a derivação gástrica em Y de Roux (DGYR) a cirurgia mais empregada (SANTO et al., 2010). As complicações relacionadas a cirurgia vêm ganhando importância pois a população está aderindo cada vez mais esse tipo de tratamento (TINOCO et al., 2002; MONKHOUSE et al., 2009).

A úlcera duodenal perfurada no segmento excluso em pacientes submetidos ao Bypass Gástrico em Y-de-Roux (BGYR) é uma complicação rara sendo encontrado na literatura apenas 21 casos bem reportados no mundo (ISKANDAR et al., 2014; GYPEN et al., 2008). MacGregor et al. (1999) através de estudo realizado afirmam que o tempo entre a cirurgia e a perfuração da úlcera duodenal pode variar entre 20 dias e 12 anos. A maioria dos casos apontam uma incidência maior no pós-operatório precoce em pacientes que não estavam em uso de Inibidores de Bomba de Prótons (IBP) (ISKANDAR et al., 2014;), já que os IBPs são responsáveis pela supressão da secreção de ácido gástrico por meio da inibição da enzima $\mathrm{H}+/ \mathrm{K}+$-ATPase da célula parietal gástrica (WANNMACHER, 2004).

Vários mecanismos têm sido usados para explicar a ulcerogênese nesse grupo de pacientes e acredita-se que a infecção por Helicobacter pylori seja o principal deles (KINI; KANNAN, 2011). O uso de antiinflamatórios não esteroides (AINE) ou consumo excessivo de álcool também são fatores preditivos. Segundo Bjorkman et al. (1989), a acidez no estômago excluso não é neutralizada pelo alimento como normalmente acontece em pacientes sem alterações anatômicas, além disso existe um atraso na 
liberação de bicarbonato, sendo a mucosa exposta ao ácido gástrico por um período prolongado e ao mesmo tempo também pelo refluxo biliar, agravando os efeitos de lesão na mucosa (BJORKMAN et al., 1989).

\section{É importante atentar-se para o} diagnóstico da úlcera duodenal perfurada nos pacientes com histórico cirúrgico de BGYR apresentando dor abdominal principalmente em abdome superior, pois os sinais e sintomas na maioria das vezes são inespecíficos (GREENSTEIN; O'ROURKE， 2011). O pneumoperitônio nem sempre é encontrado nos exames de imagem, sendo relatado em apenas um caso na literatura, segundo Charuzi et al. (1986) e Feres e Parra (2008). A tomografia computadorizada (TC) é o exame mais utilizado para o diagnóstico dessa patologia e são encontrados sinais como líquido livre no peritônio e um processo inflamatório no quadrante superior direito, além de identificar outras possíveis causas de abdome agudo cirúrgico relacionados ou não a cirurgia prévia (ISKANDAR et al., 2014). $\mathrm{Na}$ atualidade a videolaparoscopia vem ganhando um importante papel na abordagem desta patologia, pois além de possibilitar um diagnóstico precoce, também oferece a oportunidade de tratamento em mesmo procedimento.

O tratamento de urgência consiste em exploração cirúrgica e o fechamento primário da lesão pela técnica de ulcerorrafia e omentoplastia. Já o tratamento definitivo, que consiste em vagotomia e piloroplastia ou gastrectomia pode ser indicado no momento da abordagem ou posteriormente, de acordo com cada caso, devendo basear-se nos riscos específicos e os benefícios para o paciente. MacGregor et al. (1999), Em portadores de alto risco operatório, a terapia IBP a longo prazo é uma alternativa aceitável. A abordagem pode ser aberta ou laparoscópica, onde a última mostrou-se mais segura (ISKANDAR et al., 2014; PORIES, 2008). Uma questão vital para o sucesso do tratamento é a indicação da cirurgia definitiva.

\section{Conclusão}

A úlcera duodenal perfurada em segmento intestinal excluso de pacientes submetidos previamente ao Bypass Gástrico em Y-de-Roux é uma afecção rara e difícil diagnóstico. Pôde-se observar através deste caso que devemos nos atentar em pacientes que já foram submetidos a Bypass Gástrico em Y-de-Roux apresentando dor abdominal, mesmo que esta seja inespecífica e sem outros sinais ou sintomas. Além disto foi visto a opção da laparoscopia diagnóstica e terapêutica como método a ser considerado, pela eficácia apresentada.

\section{Referências}

ABESO. Associação Brasileira para o Estudo da Obesidade e da Síndrome Metabólica. Diretrizes brasileiras de obesidade 2009/2010. 3.ed. Itapevi, SP: AC Farmacêutica, 2009. 
ADAMI, G.F. et al. Metabolic syndrome in severely obese patients. Obes Surg. v. 11, n. 5, p. 543-545, Oct. 2001.

ALVAREZ-CORDERO, R. Treatment of Clinically Severe Obesity, a Public Health Problem: Introduction. World Journal of Surgery, v. 22, Issue 9, p. 905-906, September, 1998.

BJORKMAN, D.J. et al. Perforated duodenal ulcer after gastric bypass surgery. Am J Gastroenterol., v. 84, n. 2, p. 1702, Feb. 1989.

BUCHWALD, H. et al. Bariatric surgery: a systematic review and metaanalysis. JAMA, v. 293, n. 14, p. 1728, 13 Apr. 2005.

CHARUZI, I. et al. Perforation of duodenal ulcer following gastric exclusion operation for morbid obesity. J Clin Gastroenterol. v. 8, n. 5, p. 6056, Oct. 1986.

FERES, O.; PARRA, R.S. Abdômen agudo. Simpósio: Fundamentos em Clínica Cirúrgica, $2^{\mathrm{a}}$ Parte. Cap. 1. v. 41, n. 4, p. 430-6, Medicina (Ribeirão Preto) 2008.

FORCINA, D.V. et al. Papel da cirurgia bariátrica no controle do diabete

melito tipo II. ABCD Arq. Bras. Cir. Dig., v. 21, n. 3, São Paulo July/Sept. 2008

\section{GARRIDO JÚNIOR, A.B. Cirurgia da} obesidade. São Paulo: Atheneu, 2006.

GREENSTEIN, A.J.; O'ROURKE, R.W. Dor abdominal após by-pass gástrico: suspeitos \& soluções. Am J Surg. v. 6, p. 819-827, jun. 2011.

ISKANDAR, M.E. et al. Diagnosis and Management of Perforated Duodenal Ulcers following Roux-En-Y Gastric Bypass: a reporto of two cases and a review of the literature. Hindawi Publishing Corporation Case Reports in Surgery, v. 2015, Article ID 353468. Disponível em: http://www.hindawi.com/journals/cris/2015/ 353468/. Acesso em: 20 jun. 2015. KINI, S.; KANNAN, U. Effect of bariatric surgery on future general surgical procedures. J Minim Access Surg.; v. 7, n. 2, p. 126 -131, Apr./Jun, 2011.

LAMARCHE, J. Abdominal obesity and its metabolic complications: implications for the risk of ischaemic heart disease. Coron Artery Dis. v. 9, n. 8, p. 473-481, 1998.

MACGREGOR, A.M. et al. Úlcera péptica perfurada seguinte by pass gástrico para a obesidade. Am Surg Journal, v. 65, n. 3, p. 222225, mar. 1999.

MASON, E.E. et al. Effect of gastric bypass on gastric secretion. Am J Surg., v. 131, n. 2, p. 1628, Feb. 1976.

MITTERMAIR, R.; RENZ, O. An unusual complication of gastric by-pass: perforated duodenal ulcer. Obesity Surgery, v. 17, p. 701703, 2007.

MONKHOUSE, S.J.W. et al. Complications of bariatric surgery: presentation and emergency management - a review. Am R Coll Surg Engl, v. 91 , p. $280-286,2009$.

PORIES, W.J. Bariatric Surgery: Risks and Rewards. The Journal of Clinical Endocrinology \& Metabolism, v. 93, n. 11, suppl.1, 2015.

SANTO, M.A. et al. Tratamento cirúrgico da obesidade mórbida implicações gestacionais. Rev. Assoc. Med. Bras. v. 56, n. 6, São Paulo, 2010.

SIQUEIRA, J.S. et al. Aspectos gerais nas infecções por Helicobacter pylori:

Revisão. RBAC, v. 39, n. 1, p. 9-13, 2007.

SPERANZINI, M.B.; DEUTSCH, C.R.; YAGI, O.K. Manual de Diagnóstico e Tratamento para o Residente de Cirurgia. São Paulo: Atheneu, 2013, Cap. 7.

TINOCO, R.C. et al. Cirurgia da obesidade mórbida por videolaparoscopia. Rev. Col. Cir., v. 29, n. 3, maio/jun, 2002.

WANNMACHER, L. Inibidores da bomba de prótons: indicações racionais. In: Uso racional de medicamentos: temas selecionados. v. 2, n. 1 . Brasília, dez. 2004. 